\title{
Langmuir probe measurements in a time-fluctuating-highly ionized non-equilibrium cutting arc: Analysis of the electron retarding part of the time-averaged current-voltage characteristic of the probe
}

\author{
L. Prevosto, ${ }^{1, a)}$ H. Kelly, ${ }^{1,2}$ and B. Mancinelli ${ }^{1}$ \\ ${ }^{1}$ Grupo de Descargas Eléctricas, Departamento Ing. Electromecánica, Facultad Regional Venado Tuerto \\ (UTN), Laprida 651, Venado Tuerto (2600) Santa Fe, Argentina \\ ${ }^{2}$ Instituto de Física del Plasma (CONICET), Departamento de Física, Facultad de Ciencias Exactas y \\ Naturales (UBA) Ciudad Universitaria Pab. I, (1428) Buenos Aires, Argentina
}

(Received 6 August 2013; accepted 2 December 2013; published online 19 December 2013)

\begin{abstract}
This work describes the application of Langmuir probe diagnostics to the measurement of the electron temperature in a time-fluctuating-highly ionized, non-equilibrium cutting arc. The electron retarding part of the time-averaged current-voltage characteristic of the probe was analysed, assuming that the standard exponential expression describing the electron current to the probe in collision-free plasmas can be applied under the investigated conditions. A procedure is described which allows the determination of the errors introduced in time-averaged probe data due to small-amplitude plasma fluctuations. It was found that the experimental points can be gathered into two well defined groups allowing defining two quite different averaged electron temperature values. In the low-current region the averaged characteristic was not significantly disturbed by the fluctuations and can reliably be used to obtain the actual value of the averaged electron temperature. In particular, an averaged electron temperature of $0.98 \pm 0.07 \mathrm{eV}(=11400 \pm 800 \mathrm{~K})$ was found for the central core of the arc $(30 \mathrm{~A})$ at $3.5 \mathrm{~mm}$ downstream from the nozzle exit. This average included not only a time-average over the time fluctuations but also a spatial-average along the probe collecting length. The fitting of the high-current region of the characteristic using such electron temperature value together with the corrections given by the fluctuation analysis showed a relevant departure of local thermal equilibrium in the arc core. () 2013 AIP Publishing LLC. [http://dx.doi.org/10.1063/1.4848916]
\end{abstract}

\section{INTRODUCTION}

The plasma cutting process is characterized by a transferred electric arc that is established between a cathode, which is a part of the cutting torch, and a work-piece (the metal to be cut) acting as the anode. ${ }^{1}$ In order to obtain a high-quality cut, the plasma jet must be as collimated as possible (i.e., it must have high power density). To this end, the transferred arc is constricted by a metallic tube (a nozzle) with a small inner diameter (of the order of $1 \mathrm{~mm}$ ). A vortex-type high-pressure flow is forced through the nozzle to provide arc stability and to protect its inner wall. The intense convective cooling at the arc fringes due to the vortex flow enhances the power dissipation per unit length of the arc column, which in turn, results in a high axial electron temperature and ionization degree. Typically, electron temperatures of about $1 \mathrm{eV}$ for low current arcs, to about $2 \mathrm{eV}$ for higher currents; and ionization degrees of the order of $10 \%$ or higher, are usually reached. ${ }^{1}$ The arc current varies typically from ten to a few hundred of amperes, which makes an effective filtering of the power source ripple difficult. Ripple factors that vary between $5 \%$ and $10 \%$ of the mean arc voltage are a common feature in direct-current (dc) cutting torches. ${ }^{2,3}$ If a 3 -phase transductor type of power supply is used, then the fundamental ripple frequency is that of the third harmonic, and if 3-phase silicon controlled rectifier based power supply is used, then the ripple frequency matches

a)Electronic mail: prevosto@waycom.com.ar the sixth harmonic. The strong oscillatory components in the arc voltage and current will produce, in turn, large fluctuations in the plasma quantities that vary at the ripple frequency. ${ }^{2}$

The Langmuir (electrostatic) probe is an important diagnostic to obtain spatially and time-resolved information on plasma parameters, mostly in low-pressure, low-ionized plasma discharges. ${ }^{4-6}$ However, these probes have been also successfully employed to study dc arc torches operated at atmospheric pressure. ${ }^{7-14}$ In these studies the diagnostic was based on the analysis of the ion current branch of the probe characteristic curve.

The presence of fluctuations in the plasma parameters (plasma density, electron temperature, and plasma voltage) can modify the profile of the probe characteristic curve (and its derivatives), leading to substantial errors in the measurements. ${ }^{4,5}$ The effect of plasma fluctuations on the measurement of the electron distribution function by introducing the fluctuating part of the potential into an analytical model of the probe characteristic curve was previously investigated. A quantitative relation between the measurements and the true electron distribution function was found. ${ }^{15}$ The effect of the plasma fluctuations on the measurements of fluid observables (i.e., statistical moments of the electron distribution function) was considered in the work of Crawford. ${ }^{16}$ In that work, the effect of the fluctuations in the electron temperature, plasma density, and plasma voltage on the electronretarding part of the probe characteristic was considered. Such analysis is here generalized to include also the fluctuations 
in the probe voltage. This work concerns the application of Langmuir probe diagnostics to the measurement of the electron temperature in a time-fluctuating-highly ionized, nonequilibrium cutting arc, by using the electron retarding part of the current-voltage characteristic curve of the probe. Hence, the attention has been focused on the quantitative errors in the probe measurements caused by these fluctuations.

The paper is organized as follows: the experimental setup is described in Sec. II. The experimental results are given in Sec. III, while the analysis of the fluctuations affecting the retarding part of the electron branch and its results in terms of the electron temperature are presented in Sec. IV. The conclusions are summarized in Sec. V.

\section{EXPERIMENTAL SETUP}

\section{A. Arc cutting torch}

The experiment was carried out using a low-current cutting torch operated with oxygen as the plasma gas. It consisted of a cathode centered above an orifice in a converging-straight copper nozzle with liquid cooling. The cathode was made of copper ( $7 \mathrm{~mm}$ in diameter) with a hafnium tip $(1.5 \mathrm{~mm}$ in diameter) inserted at the cathode center. A flow of oxygen gas cooled the cathode and was also employed as the plasma gas. The gas passed through a swirl ring to provide arc stability. The nozzle consisted in a converging-straight bore (with a bore radius of $0.5 \mathrm{~mm}$ and a length of $4.5 \mathrm{~mm}$ ) in a copper holder surrounding the cathode (with a separation of $0.5 \mathrm{~mm}$ between the holder and the cathode surface). To avoid plasma contamination by metal vapors from the anode, a rotating steel disk was used as the anode, with its upper surface located at $5 \mathrm{~mm}$ from the nozzle exit. A well-stabilized arc column was obtained, with the arc root sliding on the disk lateral surface. It was found that this surface resulted completely not melted (thus, practically no metal vapors from the anode were present in the arc). More details on the used arc torch can be found in Ref. 7. A 3-phase transductor power supply with a main sinusoidal voltage ripple level of about $8 \%$ (peak value) and a fundamental frequency of $150 \mathrm{~Hz}$ was used to run the torch. The measured torch operating conditions were: arc current $30 \mathrm{~A}$, gas mass flow rate $0.58 \mathrm{~g} \mathrm{~s}^{-1}$, and torch chamber pressure $0.7 \mathrm{MPa}$.

\section{B. Sweeping Langmuir probe device}

The employed sweeping Langmuir probe system together with its biasing circuit is shown schematically in Fig. 1. It consisted in a rotating aluminium disk carrying one probe mounted in the radial outward direction. On one of the disc surfaces a pair of carbon brushes collected the probe current. The probes length and the disc diameter were chosen large enough in order to consider that the probe axis was approximately parallel to the line joining the jet axis to the disc centre during the whole passing of the probe through the arc. The sweep velocity of the probe was $17 \mathrm{~m} \mathrm{~s}^{-1}$ (radial sweep time of about $100 \mathrm{~ms}$, see Fig. 3). The probe was made of thin copper wires with a radius $R_{p}=150 \mu \mathrm{m}$. The low value of the copper melting temperature (lower than $1500 \mathrm{~K}$ ) rules out the

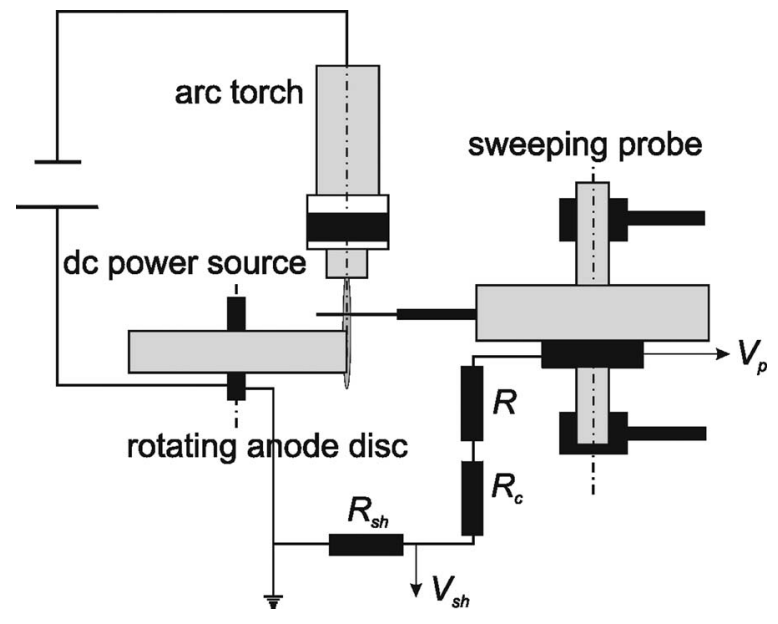

FIG. 1. Experimental setup showing the torch, the sweeping probe, and the electrical connections.

thermoelectric emission from the probe $;{ }^{17}$ however, the harsh thermal conditions in the arc prevented use of copper wires with radius lower than $150 \mathrm{~mm}$. As in several other previous experiments, ${ }^{7-9}$ the probe was located at $3.5 \mathrm{~mm}$ downstream from the torch exit.

To obtain the electron retarding part of the probe characteristic curve the probe was negatively biased employing only a resistor ( $R$, see Fig. 1). In the experiment $R$ was varied in the wide range of $0.9-1000 \Omega$. The voltage drop on a calibrated current shunt $\left(R_{s h}=0.044 \Omega\right)$ was also used to determine the probe current $\left(I_{p}\right)$ thus avoiding the effects of the straight resistance ( $R_{c}$ of about $0.1 \Omega$ ) of the probe circuit when the lower values of $R$ were used. The probe voltage $\left(V_{p}\right)$ (with respect to the grounded anode) and the voltage drop across the shunt $\left(V_{s h}\right)$ were simultaneously measured by using a twochannel oscilloscope (Tektronix TDS 1002 B with a sampling rate of $500 \mathrm{MS} / \mathrm{s}$ and an analogical bandwidth of $60 \mathrm{MHz}$ ). The probe voltage and the probe current was then related by

$$
V_{p}=-I_{p} R_{e f f}
$$

(where $R_{\text {eff }} \equiv R+R_{s h}+R_{c}$ is the effective biasing resistance, which was calculated from the measurements as $\left.R_{e f f}=R_{s h}\left(V_{p} / V_{s h}\right)\right)$.

\section{EXPERIMENTAL RESULTS}

\section{A. Theoretical considerations}

Electrostatic probes can provide information about the plasma charge density, plasma electron temperature, and plasma potential. Unfortunately, no comprehensive theory is available for the interpretation of data collected under the plasma conditions contemplated in this study (i.e., highly ionized atmospheric plasmas). ${ }^{17}$ These plasma conditions are often identified as "hybrid" in the sense that although the probe essentially operates in the diffusion regime (its dimensions being typically much larger than any mean-free-path in the plasma), the plasma shielding length (Debye length) is shorter than at least the electron mean-free-path. Under such conditions the electron collection process at the probe is essentially through a collisionless sheath. Assuming that (a) the electron 
density follows the Boltzmann distribution in near-probe region (the perturbation created by the probe current in the nearprobe region is localized in a thin near-wall layer in which the electron density deviates from the Boltzmann distribution), (b) the electron temperature is nearly constant in the nearprobe region, and (c) the probe operates in the moderately retarding regime (therefore, at negative potential with respect to the plasma); the electron current collected by the probe is described by the same formula as in the theory of Langmuir probes in collision-free plasmas: ${ }^{17-19}$

$$
I_{e}=e \frac{n_{e} \bar{v}_{e}}{4} A \exp \left(e \frac{V_{p}-V_{s}}{k T_{e}}\right)
$$

( $e$ is the electron charge, $n_{e}$ is the electron density far from the probe, $\bar{v}_{e}$ is the electron mean velocity, $A$ is the collecting area of the probe $\left(=2 \pi R_{p} L_{p}\right.$ in this cylindrical case, with $L_{p}$ being its length), $T_{e}$ is the electron temperature far from the probe, $V_{s}$ is the plasma potential, and $k$ is the Boltzmann constant). The electron temperature can then be obtained from Eq. (2) as

$$
\frac{k T_{e}}{e}=\left(\frac{1}{I_{e}} \frac{\partial I_{e}}{\partial V_{p}}\right)^{-1} .
$$

Several works have experimentally demonstrated the reliability of Eq. (3) for the determination of the electron temperature of atmospheric pressure plasmas. ${ }^{19-22}$

In Fig. 2 plots of the mean-free-path for elastic collisions between electrons and heavy particles, $\lambda_{\text {emfp }}$, of the meanfree-path for elastic collisions between electrons and neutral particles, $\lambda_{e a}$, of the electron Debye length, $\lambda_{D}$, of the characteristic length of the thermal layer, $\delta$, of the relaxation length of the electron energy in elastic collisions with heavy particles, $\lambda_{u}$, and of the characteristic length of the near-wall layer in which the electron density deviates from the Boltzmann distribution, $y_{B}$; estimated for the conditions in the unperturbed non-equilibrium, atmospheric pressure, oxygen plasma, as function of $T_{e}$, are shown.

$\left(\lambda_{\text {emfp }} \equiv\left(n Q^{e, 0}+n_{i} Q^{e,+}\right)^{-1}, Q^{e, 0}\right.$ and $Q^{e,+}$ being the electron-neutral and electron-ion collision crosssections, $n_{i}$ being the positive ion density, and $n$ being the neutral particles density; respectively; $\lambda_{e a} \equiv\left(n Q^{e, 0}\right)^{-1}$; $\lambda_{D} \equiv \sqrt{\varepsilon_{0} T_{e} / n_{e} e^{2}}, \varepsilon_{0}$ being the permittivity of the freespace; $\delta^{-1} \approx 9 / 4(2 m / M) n_{e} v_{e} k R_{p} \theta / \kappa_{h},{ }^{10} m$ being the electron mass, $M$ being the heavy particles mass, $v_{e}$ being the frequency of the electron-heavy particle collisions,

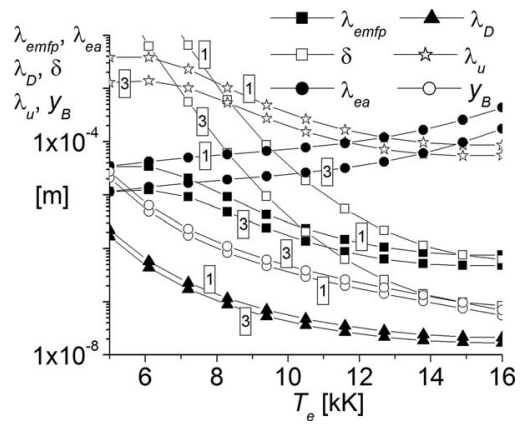

FIG. 2. Characteristic lengths in atmospheric pressure oxygen plasma vs. electron temperature. For the symbols see the text. and $\kappa_{h}$ being the heavy particle thermal conductivity; $\lambda_{u}$ $\equiv \lambda_{\text {emfp }}(M / 2 m)^{1 / 2} ;$ and $y_{B} \equiv\left(\lambda_{D}^{2} R_{p} n Q^{i, 0}\right)^{1 / 2},^{18} Q^{i, 0}$ being the ion-neutral cross-section for elastic collisions). The nonequilibrium plasma composition was calculated using the generalized Saha equation, ${ }^{23}$ together with the equation of state. It should be noted that although the employed gas carrier was oxygen in this work, the presence of negative molecular or atomic ions can be neglected owing to the high electron temperature reached in this kind of arcs. ${ }^{24}$ The elastic cross-section data used for these calculations were taken from Ref. 17. The value of the ratio $\theta \equiv T_{e} / T_{h}\left(T_{h}\right.$ is the heavy particles temperature) was indicated by a label in each curve. In particular, $\theta=1$ corresponds to a local thermodynamic equilibrium (LTE) plasma, while $\theta=3$ describes a strong deviation from LTE.

As it can be seen from Fig. 2, the sheath is collisionless for electrons in the whole considered $T_{e}$ range (i.e., from a weakly to highly ionized plasma). For $T_{e}$ higher than about $10000 \mathrm{~K}$, the electron cooling in the plasma region perturbed by the probe can be neglected since its relaxation energy length remains much higher than the extent of the thermal layer in which the gas temperature is depleted to a value close to the probe-wall temperature. ${ }^{10}$ For the same $T_{e}$ range (i.e., for a highly ionized plasma), also the thickness of the near-wall layer in which the electron density deviates from the Boltzmann distribution is much smaller than the electron mean-free-path for collisions with neutral particles (near the wall the ion density is very low as compared with the unperturbed density and hence there the electrons mainly collide with neutral particles). It should be noted that the electron density in the plasma region around the probe satisfies the Boltzmann distribution due to the smallness of the net electron flux (the term "smallness" means that the net electron flux is much smaller than the fluxes caused by the electron pressure gradient and by the electric field; but is not necessarily small as compared to the ion flux). An important conclusion derived from Fig. 2 is that only for highly ionized plasmas, the "standard" formula given by Eq. (2) can be used in high-pressure plasmas. In high-pressure weakly ionized plasma conditions (as the diffusive regime considered in Refs. 4 and 5), formula (2) is not valid because the assumption (a) is not fully accomplished (Eq. (35) of Ref. 4 should be used instead); although the electron temperature still can be estimated with a limited degree of accuracy on the basis of the slope of the electron retarding of the probe characteristic curve (see discussion in Sec. 6.8.4 of Ref. 17). Experimental evidence supporting that the electrons retain its Boltzmann distribution throughout the near-probe region will be given later (see Fig. 5).

\section{B. Averaged current-voltage curve}

Due to the presence of plasma fluctuations it was necessary to average each point of the probe current-voltage characteristic curve over many probe sweeps in order to obtain a smooth probe characteristic curve. In the present case 128 probe signals were averaged for each probe condition using the 128 times $(128 \times)$ average acquisition mode of the 


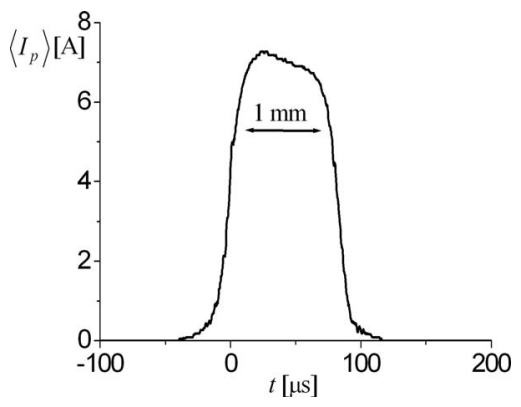

FIG. 3. Averaged probe current sample for $R_{\text {eff }}=0.99 \Omega$. The corresponding spatial scale based on the probe velocity is also shown.

oscilloscope. Since the probe takes a time of about $5 \mathrm{~s}$ to traverse 128 times the arc, and this time is much larger than the typical fluctuation time-scale (of about $7 \mathrm{~ms}$ ); this averaging mode almost quenched the arc fluctuations. It should be noted that the probe transit time trough the arc (of about $100 \mu \mathrm{s}$ ) was much shorter than the time-scale of the plasma fluctuations and so each probe sample corresponds to a practically constant value of the plasma voltage (which varied in $\pm 8 \%$ around of a mean voltage value).

A typical $(128 \times \mathrm{X})$ averaged current waveform $\left\langle I_{p}\right\rangle$ corresponding to an effective biasing resistance $R_{\text {eff }}=0.99 \Omega$ is shown in Fig. 3. It can be seen that this signal appears with a Gaussian-like shape after the quenching of the plasma fluctuation effects. A spatial-scale (based on the probe sweeping velocity) is also shown in Fig. 3.

Using the central value of the averaged probe signals, the electron retarding part of the averaged current-voltage characteristic probe was built. This was done experimentally by varying the $R$ value in the probe circuit (see Fig. 1), since each selected value of $R$ produces a specific value of $\left\langle I_{p}\right\rangle$. The obtained results are given in Fig. 4.

To derive the electron temperature from the probe characteristic, it is necessary to know the ion current contribution. In previous experiments with the same torch, ${ }^{7,8}$ but measuring the ion current to the probe (well below the floating potential value), it was found that the ion current did not saturate, but grew proportional to the electric field value at the vicinities of the probe. If this collecting model could be extrapolated to probe voltages larger than the floating value, this will give an indication that the ion current decreases continuously for increasing probe voltages (see Eqs. (10) and (11) of Ref. 8). In any case, in the absence of a reliable ion collection model for

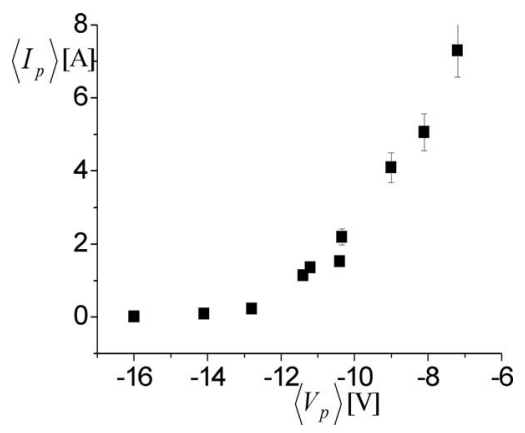

FIG. 4. Averaged probe characteristic curve.

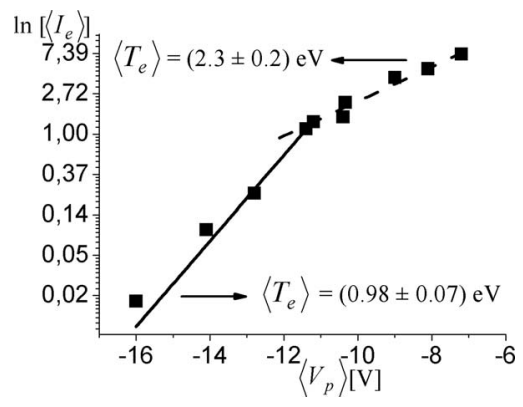

FIG. 5. Averaged electronic current in semi-log scale as a function of the averaged probe voltage. The dashed line is the least square fit of the large electron current points, while the solid one is the fit of the small electron current points.

probe voltages larger than the floating value, it was assumed that the ion contribution in the electron branch is negligible as compared to the electron one, $\left\langle I_{e}\right\rangle \approx\left\langle I_{p}\right\rangle$. The consistency of this assumption will be checked later (using the model predictions) by calculating the electron averaged current from Eq. (2) and comparing these obtained values with the experimental $\left\langle I_{p}\right\rangle$ data.

The dependence of the averaged electron current $\left\langle I_{e}\right\rangle$ on the probe potential plotted on a semi-logarithmic scale is showed in Fig. 5. It can be seen that the experimental points can be gathered into two well defined groups allowing defining two quite different $\left\langle T_{e}\right\rangle$ values. It should be noted that $\left\langle T_{e}\right\rangle$ represents a certain spatial-average along the effective collecting length of the probe, which from the spatial-scale shown in Fig. 3 can be estimated as $1 \mathrm{~mm}$. The dashed line in Fig. 5, corresponding to the large current group, yields $\left\langle T_{e}\right\rangle$ of about $2.3 \mathrm{eV}$; which is an unreliable large value for low-current cutting arcs. The solid line in Fig. 5, corresponding to the low current group, yields $\left\langle T_{e}\right\rangle$ of about $1 \mathrm{eV}$; which lies in the typical range of $\left\langle T_{e}\right\rangle$ for low-current cutting arcs., $8,25,26$ An explanation on such a probe characteristic trend in terms of the errors introduced in the probe measurements by the plasma fluctuations will be presented in Sec. IV.

\section{INTERPRETATION OF THE AVERAGED DATA}

\section{A. Plasma fluctuations analysis}

If fluctuating components of $I_{e}, n_{e}, T_{e}, V_{p}$, and $V_{s}$ are taken into account in the general expression

$$
I_{e}=f\left(n_{e}, T_{e}, V_{p}, V_{s}\right)
$$

the electron current to the probe can be expressed as

$$
\left\langle I_{e}\right\rangle+I_{e}^{\prime}=f\left(\left\langle n_{e}\right\rangle+n_{e}^{\prime},\left\langle T_{e}\right\rangle+T_{e}^{\prime},\left\langle V_{p}\right\rangle+V_{p}^{\prime},\left\langle V_{s}\right\rangle+V_{s}^{\prime}\right),
$$

where the comma indicates a fluctuating part with zero timeaverage. Assuming that the fluctuating component is small as compared to the averaged one, Eq. (5) can be expanded by 
Taylor's theorem to give

$$
\begin{aligned}
\left\langle I_{e}\right\rangle+I_{e}^{\prime}= & I_{e}\left(\left\langle T_{e}\right\rangle,\left\langle n_{e}\right\rangle,\left\langle V_{p}\right\rangle,\left\langle V_{s}\right\rangle\right)+\left\{\frac{\partial I_{e}}{\partial T_{e}} T_{e}^{\prime}+\frac{\partial I_{e}}{\partial n_{e}} n_{e}^{\prime}+\frac{\partial I_{e}}{\partial V_{p}} V_{p}^{\prime}+\frac{\partial I_{e}}{\partial V_{s}} V_{s}^{\prime}\right\} \\
& +\frac{1}{2}\left\{\frac{\partial^{2} I_{e}}{\partial T_{e}^{2}}\left(T_{e}^{\prime}\right)^{2}+\frac{\partial^{2} I_{e}}{\partial n_{e}^{2}}\left(n_{e}^{\prime}\right)^{2}+\frac{\partial^{2} I_{e}}{\partial V_{p}^{2}}\left(V_{p}^{\prime}\right)^{2}+\frac{\partial^{2} I_{e}}{\partial V_{s}^{2}}\left(V_{s}^{\prime}\right)^{2}\right\} \\
& +\left\{\frac{\partial^{2} I_{e}}{\partial T_{e} \partial n_{e}} T_{e}^{\prime} n_{e}^{\prime}+\frac{\partial^{2} I_{e}}{\partial T_{e} \partial V_{s}} T_{e}^{\prime} V_{s}^{\prime}+\frac{\partial^{2} I_{e}}{\partial T_{e} \partial V_{p}} T_{e}^{\prime} V_{p}^{\prime}+\frac{\partial^{2} I_{e}}{\partial n_{e} \partial V_{s}} n_{e}^{\prime} V_{s}^{\prime}\right. \\
& \left.+\frac{\partial^{2} I_{e}}{\partial n_{e} \partial V_{p}} n_{e}^{\prime} V_{p}^{\prime}+\frac{\partial^{2} I_{e}}{\partial V_{s} \partial V_{p}} V_{s}^{\prime} V_{p}^{\prime}\right\},
\end{aligned}
$$

where all the $I_{e}$ derivatives are calculated at the averaged values of $n_{e}, T_{e}, V_{p}$, and $V_{s}$, and terms higher than the second order have been neglected. Taking the time-average of Eq. (6),

$$
\begin{aligned}
\left\langle I_{e}\right\rangle= & I_{e}\left(\left\langle T_{e}\right\rangle,\left\langle n_{e}\right\rangle,\left\langle V_{p}\right\rangle,\left\langle V_{s}\right\rangle\right) \\
& +\frac{1}{2}\left\{\frac{\partial^{2} I_{e}}{\partial T_{e}^{2}}\left\langle\left(T_{e}^{\prime}\right)^{2}\right\rangle+\frac{\partial^{2} I_{e}}{\partial n_{e}^{2}}\left\langle\left(n_{e}^{\prime}\right)^{2}\right\rangle+\frac{\partial^{2} I_{e}}{\partial V_{p}^{2}}\left\langle\left(V_{p}^{\prime}\right)^{2}\right\rangle+\frac{\partial^{2} I_{e}}{\partial V_{s}^{2}}\left\langle\left(V_{s}^{\prime}\right)^{2}\right\rangle\right\} \\
& +\left\{\frac{\partial^{2} I_{e}}{\partial T_{e} \partial n_{e}}\left\langle T_{e}^{\prime} n_{e}^{\prime}\right\rangle+\frac{\partial^{2} I_{e}}{\partial T_{e} \partial V_{s}}\left\langle T_{e}^{\prime} V_{s}^{\prime}\right\rangle+\frac{\partial^{2} I_{e}}{\partial T_{e} \partial V_{p}}\left\langle T_{e}^{\prime} V_{p}^{\prime}\right\rangle+\frac{\partial^{2} I_{e}}{\partial n_{e} \partial V_{s}}\left\langle n_{e}^{\prime} V_{s}^{\prime}\right\rangle\right. \\
& \left.+\frac{\partial^{2} I_{e}}{\partial n_{e} \partial V_{p}}\left\langle n_{e}^{\prime} V_{p}^{\prime}\right\rangle+\frac{\partial^{2} I_{e}}{\partial V_{s} \partial V_{p}}\left\langle V_{s}^{\prime} V_{p}^{\prime}\right\rangle\right\} \equiv I_{e}\left(\left\langle T_{e}\right\rangle,\left\langle n_{e}\right\rangle,\left\langle V_{p}\right\rangle,\left\langle V_{s}\right\rangle\right)+\tilde{I}_{e} .
\end{aligned}
$$

It is seen from Eq. (7) that there is an additional current term $\left(\tilde{I}_{e}\right)$ related to the presence of fluctuations. The first bracket of it contains the variance values of the fluctuations, while the second one contains its cross-correlations. Since that the electron current is given by Eq. (2) (the plasma fluctuations do not cause excursion into the electron saturation current region), the additional current term in Eq. (7) can be written finally as

$$
\begin{aligned}
\tilde{I}_{e}= & I_{e}\left(\left\langle T_{e}\right\rangle,\left\langle n_{e}\right\rangle,\left\langle V_{p}\right\rangle,\left\langle V_{s}\right\rangle\right)\left[\left\{\left(-\frac{1}{4}+\frac{e\left(\left\langle V_{p}\right\rangle-\left\langle V_{s}\right\rangle\right)}{k\left\langle T_{e}\right\rangle}+\left(\frac{e\left(\left\langle V_{p}\right\rangle-\left\langle V_{s}\right\rangle\right)}{k\left\langle T_{e}\right\rangle}\right)^{2}\right)\left\langle\left(\frac{T_{e}^{\prime}}{\left\langle T_{e}\right\rangle}\right)^{2}\right\rangle\right.\right. \\
& \left.+\frac{1}{2}\left\langle\left(\frac{e V_{p}^{\prime}}{k\left\langle T_{e}\right\rangle}\right)^{2}\right\rangle+\frac{1}{2}\left\langle\left(\frac{e V_{s}^{\prime}}{k\left\langle T_{e}\right\rangle}\right)^{2}\right\rangle\right\} \frac{1}{2}+\left(\frac{1}{2}-\frac{e\left(\left\langle V_{p}\right\rangle-\left\langle V_{s}\right\rangle\right)}{k\left\langle T_{e}\right\rangle}\right)\left\langle\frac{T_{e}^{\prime}}{\left\langle T_{e}\right\rangle} \frac{n_{e}^{\prime}}{\left\langle n_{e}\right\rangle}\right\rangle \\
& +\left(\frac{1}{2}+\frac{e\left(\left\langle V_{p}\right\rangle-\left\langle V_{s}\right\rangle\right)}{k\left\langle T_{e}\right\rangle}\right)\left(\left\langle\frac{T_{e}^{\prime}}{\left\langle T_{e}\right\rangle} \frac{e V_{s}^{\prime}}{k\left\langle T_{e}\right\rangle}\right\rangle-\left\langle\frac{T_{e}^{\prime}}{\left\langle T_{e}\right\rangle} \frac{e V_{p}^{\prime}}{k\left\langle T_{e}\right\rangle}\right\rangle\right)-\left\langle\frac{n_{e}^{\prime}}{\left\langle n_{e}\right\rangle} \frac{e V_{s}^{\prime}}{k\left\langle T_{e}\right\rangle}\right\rangle \\
& \left.+\left\langle\frac{n_{e}^{\prime}}{\left\langle n_{e}\right\rangle} \frac{e V_{p}^{\prime}}{k\left\langle T_{e}\right\rangle}\right\rangle-\left\langle\frac{e V_{p}^{\prime}}{k\left\langle T_{e}\right\rangle} \frac{e V_{s}^{\prime}}{k\left\langle T_{e}\right\rangle}\right\rangle\right] .
\end{aligned}
$$

The probe current has influence on the voltage of the probe as it was biased to the anode trough a resistor (see Fig. 1). From Eq. (1), the corresponding additional term to the probe voltage is given by

$$
\tilde{V}_{p} \equiv-R_{e f f} \tilde{I}_{e}
$$

where

$$
\left\langle V_{p}\right\rangle \equiv V_{p}\left(\left\langle T_{e}\right\rangle,\left\langle n_{e}\right\rangle,\left\langle V_{s}\right\rangle, R_{e f f}\right)+\tilde{V}_{p} .
$$

In order to evaluate $\tilde{I}_{e}$ and $\tilde{V}_{p}$ (see Eqs. (8) and (9)), it is necessary to know the fluctuating part of the plasma variables. For the plasma voltage it was adopted the expression

$$
V_{s}^{\prime}=\left\langle V_{s}\right\rangle \zeta(t)
$$

with $\zeta(t)$ being a dimensionless function characterizing plasma voltage fluctuations. Furthermore,

$$
T_{e}^{\prime}=\left\langle T_{e}\right\rangle \xi(t)
$$

with $\xi(t)$ being a dimensionless function characterizing electron temperature fluctuations. The voltage fluctuations have a direct influence on current fluctuations, which in turn, produce fluctuations in the magnetic pressure. As the magnetic pressure effects are negligible in cutting arcs, the voltage fluctuations have also negligible effects on the arc pressure (which is close to the atmospheric value). So the pressure fluctuations were neglected in this study $\left(p^{\prime} \approx 0\right)$.

To evaluate the electron density fluctuations, a connection between the electron density and the electron 
temperature fluctuations may be found from the generalized Saha equation, ${ }^{23}$

$$
\frac{n_{e} n_{i}}{n}=2 \frac{Q_{+}}{Q_{0}}\left(\frac{2 \pi m k T_{e}}{h^{2}}\right)^{3 / 2} \exp \left(-\frac{E_{I}}{k T_{e}}\right),
$$

plus the equation of state,

$$
\frac{p}{k}=\frac{T_{e}}{\theta}\left(n_{e}(1+\theta)+n\right),
$$

and the quasi-neutrality plasma condition,

$$
n_{i} \approx n_{e}
$$

( $Q_{+}$and $Q_{0}$ are the statistical weights of atomic ions and atoms, respectively; $h$ is the Planck's constant, $E_{I}$ is the first ionization energy of the atoms). For small values of $T_{e}^{\prime} /\left\langle T_{e}\right\rangle$, the fluctuating part of electron density is given by

$$
n_{e}^{\prime} \approx \frac{\partial n_{e}}{\partial T_{e}} T_{e}^{\prime}+\frac{1}{2} \frac{\partial^{2} n_{e}}{\partial T_{e}^{2}}\left\{\left(T_{e}^{\prime}\right)^{2}-\left\langle\left(T_{e}^{\prime}\right)^{2}\right\rangle\right\},
$$

while the averaged value is given by

$$
\left\langle n_{e}\right\rangle \approx n_{e}\left(\left\langle T_{e}\right\rangle\right)+\frac{1}{2} \frac{\partial^{2} n_{e}}{\partial T_{e}^{2}}\left\langle\left(T_{e}^{\prime}\right)^{2}\right\rangle .
$$

To obtain the fluctuating part of the probe voltage, Eq. (1) that relates the fluctuating probe voltage with the fluctuating probe current (or the fluctuating electron current) was used. The fluctuating electron current was obtained from Eq. (2), whose exponential term was approximated with negligible error within the whole experimental $\left\langle V_{p}\right\rangle$ range, by a high-order rational-interpolation. Hence, an algebraic equation for $V_{p}$ was obtained. The Taylor's theorem up to the second order approximation was then applied, and a lengthy expression for $V_{p}^{\prime}$ was obtained once $\left\langle V_{p}\right\rangle$ was subtracted from $V_{p}$ :

$$
V_{p}^{\prime}=f\left(\left\langle T_{e}\right\rangle,\left\langle n_{e}\right\rangle,\left\langle V_{s}\right\rangle, R_{e f f}, T_{e}^{\prime}, n_{e}^{\prime}, V_{s}^{\prime}\right) .
$$

The fluctuations analysis is now closed provided that the dimensionless functions $\zeta(t)$ and $\xi(t)$ are known.

Note that the averaged $(128 \times)$ measured quantities can be identified with $\left\langle I_{p}\right\rangle$ and $\left\langle V_{p}\right\rangle$ in the above described fluctuation analysis. But the "true" averaged probe characteristic should be built using the $V_{p}\left(\left\langle T_{e}\right\rangle,\left\langle n_{e}\right\rangle,\left\langle V_{s}\right\rangle, R_{e f f}\right)$ and $I_{e}\left(\left\langle T_{e}\right\rangle,\left\langle n_{e}\right\rangle,\left\langle V_{p}\right\rangle,\left\langle V_{s}\right\rangle\right)$. Hence, the experimental data should be corrected by subtracting $\tilde{I}_{e}$ and $\tilde{V}_{p}$ to $\left\langle I_{p}\right\rangle$ and $\left\langle V_{p}\right\rangle$, respectively.

\section{B. Determination of the electron temperature}

As several measurements on cutting arcs have showed, ${ }^{2,3}$ the arc voltage ripple produce fluctuations of the same level on the electron temperature of the arc that vary at the ripple frequency. Furthermore, as for low-current arcs the slope of the current-voltage characteristic curve is negative, and it is expected that $T_{e}$ follows the arc current trend, it was assumed that

$$
\xi(t) \approx-\zeta(t)=\psi \operatorname{Sin}(2 \pi f t)
$$

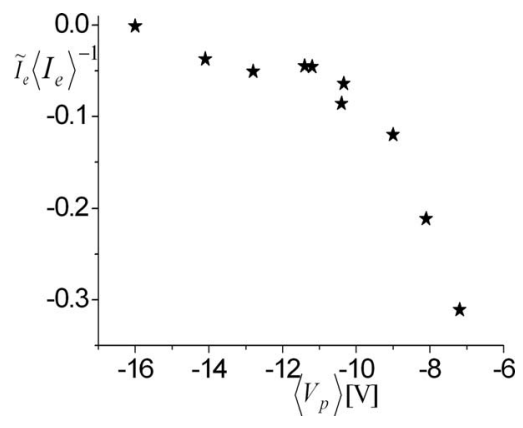

FIG. 6. Ratio $\tilde{I}_{e} /\left\langle I_{e}\right\rangle$ vs. the averaged probe voltage values $\left(\left\langle T_{e}\right\rangle=12000 \mathrm{~K}\right.$ and $\theta=1.5$ ).

where $\psi$ is the voltage ripple factor and $f$ is its main frequency. In the present case: $\psi \approx 0.08$ and $f \approx 150 \mathrm{~Hz}$ from the experimental arc voltage waveform.

A clear advantage of the Langmuir probes over other plasma diagnostic techniques in non-fluctuating plasmas (provided that Eq. (2) applies) is a straightforward determination of the electron temperature. This advantage is not longer present for the conditions studied here. The computation of the corrected probe characteristic requires the knowledge of the plasma composition. Provided that $p$ matches the atmospheric value, both $\left\langle T_{e}\right\rangle$ and $\theta$ must be initially supposed. Therefore, an iterative calculation process is necessary. The aim of such a process is to find the particular values of $\left\langle T_{e}\right\rangle$ and $\theta$ that allow to obtain a characteristic which yields the guessed value of $\left\langle T_{e}\right\rangle$. However, Fig. 5 shows that for the large negative potentials the probe characteristic yields $\left\langle T_{e}\right\rangle$ of about $1 \mathrm{eV}$, thus suggesting that close to the floating potential the characteristic is not significantly disturbed and can more reliably be used to determine the electron temperature. Guided for this value of $\left\langle T_{e}\right\rangle$, the correction values for $\left\langle I_{e}\right\rangle$ and $\left\langle V_{p}\right\rangle\left(\tilde{I}_{e}\right.$ and $\tilde{V}_{p}$, given by Eqs. (8) and (9), respectively) were calculated for a non-LTE atmospheric pressure oxygen plasma for $\left\langle T_{e}\right\rangle=12000 \mathrm{~K}$ and $\theta=1.5$ (evidence of LTE departures in this arc was previously reported by some of the authors). ${ }^{25}$ The plasma potential was taken as $-4 \mathrm{~V}$, according to previous measurements. ${ }^{7-9}$

In Fig. 6 the ratio $\tilde{I}_{e} /\left\langle I_{e}\right\rangle$ (which is the same as $\tilde{V}_{p} /\left\langle V_{p}\right\rangle$ ) is shown in the whole experimental range of the probe voltage. As it can be seen, for the large negative values of $\left\langle V_{p}\right\rangle$, the effect of plasma fluctuations is relatively small $(\leq 5 \%)$; thus supporting the hypothesis that the characteristic is not significantly disturbed close to the floating potential, and can more reliably be used to determine $\left\langle T_{e}\right\rangle$.

The original averaged probe characteristic curve as well as the corrected one is shown in semi-log scale in Fig. 7 (for $\left\langle T_{e}\right\rangle=12000 \mathrm{~K}$ and $\theta=1.5$ ). It is observed that the whole corrected data (covering a current range of three orders of magnitude) fall within a straight line which predicts $\left\langle T_{e}\right\rangle$ $=1.06 \pm 0.06 \mathrm{eV}(=12300 \pm 700 \mathrm{~K})$. This value (within the error) agrees with the electron temperature of $0.98 \pm 0.07 \mathrm{eV}$ $(=11400 \pm 800 \mathrm{~K})$ derived from the original averaged data close to the floating potential. The uncertainty in the $\left\langle T_{e}\right\rangle$ value comes from the least-square-method used to fit the data.

Note that the direct derivation of the electron temperature from the original averaged points (from the slope of 


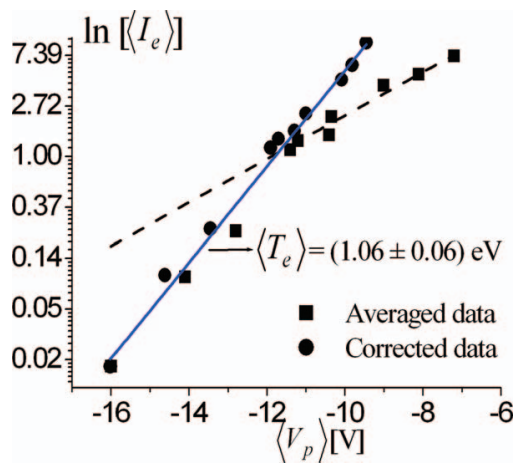

FIG. 7. Averaged and corrected probe characteristic curves. The corrections were calculated for $\left\langle T_{e}\right\rangle=12000 \mathrm{~K}$ and $\theta=1.5$.

the characteristic in the region close to the floating potential value) allows a univocal determination of $\theta$ through the rest of the procedure; that is, using Eqs. (13)-(15) together with the characteristic fitting in a region far from the floating potential value with the previously obtained electron temperature. In this way, there is no free parameter in the problem. In particular, in our measurements the value $\theta=1.5 \pm 0.2$ allows the best fitting of the characteristic in the strongly perturbed region. The uncertainty in the last value comes from different tested $\theta$ values which gave a similar (within its own uncertainty) $\left\langle T_{e}\right\rangle$ value.

Concerning to the consistency of the assumption that the ion contribution in the electron branch is negligible as compared to that of the electron, the calculation of the electron current from Eq. (2) (for $\left\langle T_{e}\right\rangle=12000 \mathrm{~K}$ and $\theta=1.5$ ) has shown that the experimental $\left\langle I_{p}\right\rangle$ data differ from $\left\langle I_{e}\right\rangle$ in less than $1 \%$ (i.e., within the experimental error); showing that for probe voltages larger than the floating one the ion current is at least two orders of magnitude lower than the electron current.

\section{CONCLUSIONS}

Electrostatic (Langmuir) probe measurements of the electron current in the retarding part of the time-averaged current-voltage characteristic in the central core of a timefluctuating-highly ionized, non-equilibrium low-current cutting arc were performed. The analysis of these measurements allowed deriving an averaged electron temperature. This average included not only a time-average over the fluctuations, but also a spatial-average along the probe collecting length. The interpretation of the probe data was based on the standard exponential relationship describing the electron retarding part of the probe characteristic in collision-free plasmas. A generalized treatment of the effects of the small-amplitude plasma fluctuations in the time-averaged probe characteristic was carried-out.

The fluctuations introduce errors into Langmuir probe measurements. It was found that the experimental points can be gathered into two well defined groups allowing defining two quite different averaged electron temperature values. In particular, a large overestimation (of about 200\%) in the electron temperature was found in the high-current region of the characteristic. On the other hand, in the low-current region the averaged characteristic was not significantly disturbed and can more reliably be used to obtain the actual value of the averaged electron temperature.

An averaged electron temperature of $0.98 \pm 0.07 \mathrm{eV}$ $(=11400 \pm 800 \mathrm{~K})$ was found for the central core of the arc (30 A) through the slope of the original averaged experimental data for the low-current region of characteristic. Taking into account that this value corresponds to a spatial-averaged electron temperature $(\approx 1 \mathrm{~mm}$ in this case), we consider this result to be in agreement with several other electron temperature determinations previously carried-out in our laboratory. The fitting of the high-current region of the characteristic using such electron temperature value together with the corrections given by the fluctuation analysis gives $\theta=1.5 \pm 0.2$, which indicates a relevant LTE departure in the arc core. Similar values for these departures were previously found using a schlieren technique.

\section{ACKNOWLEDGMENTS}

This work was supported by grants from the CONICET (PIP 112/200901/00219) and Universidad Tecnológica Nacional (PID-UTN 1389). H.K. and L.P. are members of the CONICET.

${ }^{1}$ V. A. Nemchinsky and W. S. Severance, J. Phys. D 39, R423 (2006).

${ }^{2}$ L. Prevosto, H. Kelly, and B. Mancinelli, J. Appl. Phys. 110, 083302 (2011).

${ }^{3}$ C. Pardo, J. González-Aguilar, A. Rodríguez-Yunta, and M. A. G. Calderón, J. Phys. D 32, 2181 (1999).

${ }^{4}$ V. I. Demidov, S. V. Ratynskaia, and K. Rypdal, Rev. Sci. Instrum. 73, 3409 (2002).

${ }^{5}$ V. A. Godyak and V. I. Demidov, J. Phys. D 44, 233001 (2011).

${ }^{6}$ N. St. J. Braithwaite and R. N. Franklin, Plasma Sources Sci. Technol. 18, 014008 (2009).

${ }^{7}$ L. Prevosto, H. Kelly, and B. Mancinelli, IEEE Trans. Plasma Sci. 36, 263 (2008).

${ }^{8}$ L. Prevosto, H. Kelly, and F. O. Minotti, IEEE Trans. Plasma Sci. 36, 271 (2008).

${ }^{9}$ L. Prevosto, H. Kelly, and B. Mancinelli, IEEE Trans. Plasma Sci. 37, 1092 (2009).

${ }^{10}$ L. Prevosto, H. Kelly, and B. R. Mancinelli, J. Appl. Phys. 112, 063302 (2012).

${ }^{11}$ A. E. F. Gick, M. B. C. Quigley, and P. H. Richards, J. Phys. D 6, 1941 (1973).

${ }^{12}$ C. Fanara and I. M. Richardson, J. Phys. D 34, 2715 (2001).

${ }^{13}$ C. Fanara, IEEE Trans. Plasma Sci. 33, 1072 (2005).

${ }^{14}$ C. Fanara, IEEE Trans. Plasma Sci. 33, 1082 (2005).

${ }^{15}$ A. B. Blagoev, V. I. Demidov, N. B. Kolokolov, and O. G. Toronov, Sov. Phys. Tech. Phys. 26, 1179 (1981).

${ }^{16}$ F. W. Crawford, J. Appl. Phys. 34, 1897 (1963).

${ }^{17}$ Y. P. Raizer, Gas Discharge Physics (Springer, Berlin, Germany, 1991).

${ }^{18}$ M. S. Benilov, J. Phys. D 33, 1683 (2000).

${ }^{19}$ E. Leveroni and E. Pfender, Rev. Sci. Instrum. 60, 3744 (1989).

${ }^{20}$ G. Yang, P. Cronin, J. V. Heberlein, and E. Pfender, J. Phys. D 39, 2764 (2006).

${ }^{21}$ H. E. Porteanu, S. Kühn, and R. Gesche, J. Appl. Phys. 108, 013301 (2010).

${ }^{22}$ A. Shashurin, J. Li, T. Zhuang, M. Keidar, and I. I. Beilis, Phys. Plasmas 18, 073505 (2011).

${ }^{23}$ M. C. M. van de Sanden, P. P. J. M. Schram, A. G. Peeters, J. A. M. van der Mullen, and G. M. W. Kroesen, Phys. Rev. A 40, 5273 (1989).

${ }^{24}$ M. Boulos, P. Fauchais, and E. Pfender, Thermal Plasmas, Fundamentals, and Applications (Plenum, New York, 1994), vol. 1.

${ }^{25}$ L. Prevosto, G. Artana, H. Kelly, and B. Mancinelli, J. Appl. Phys. 109, 063302 (2011).

${ }^{26}$ L. Prevosto, G. Artana, B. Mancinelli, and H. Kelly, J. Appl. Phys. 107, 023304 (2010). 